\title{
Surface composition of the largest dwarf planet 136199 Eris (2003 UB 313 $)$
}

\author{
C. Dumas ${ }^{1}$, F. Merlin ${ }^{2}$, M. A. Barucci ${ }^{2}$, C. de Bergh ${ }^{2}$, O. Hainault ${ }^{1}$, A. Guilbert ${ }^{2}$, \\ P. Vernazza ${ }^{2}$, and A. Doressoundiram ${ }^{2, \star}$ \\ 1 ESO, Alonso de Cordova 3107, Vitacura, Casilla 19001, Santiago 19, Chile \\ e-mail: cdumas@eso.org \\ 2 LESIA, Observatoire de Paris, 92195 Meudon Principal Cedex, France \\ e-mail: frederic.merlin@obspm.fr
}

Received 31 October 2006 / Accepted 1 June 2007

ABSTRACT

\begin{abstract}
Aims. The surface composition of the largest TNO, the dwarf planet 136199 Eris, is studied and compared to Pluto's. Methods. High signal-to-noise visible and near-infrared reflectance spectra were obtained at the TNG and ESO-VLT observatories. The nature and properties of the compounds present on the surface of Eris are investigated by applying Hapke and Shkuratov radiative transfer models to our spectra.

Results. The surface of Eris can be modeled using two areas of distinct composition: about $50 \%$ appears to be covered with pure methane ice, while the rest of its surface would be made of an intimate mixture of methane, nitrogen and water ices, and ice tholin. The use of nitrogen in our model is shown to improve significantly the data fit, in particular for high surface albedo values. The icy grains are found to be large, from sub-mm to a few tens of $\mathrm{mm}$ in size.
\end{abstract}

Key words. Kuiper Belt - techniques: spectroscopic - astrochemistry - infrared: solar system

\section{Introduction}

Within the last decade, the discovery of a new population of small planetary bodies, the transneptunian objects (TNOs), orbiting the Sun beyond the distance of Neptune (e.g. Jewitt \& Luu 1993; Luu et al. 1997), and the study of their dynamical properties (e.g. Jewitt et al. 1998), demonstrated that Pluto, the furthest out planet in our solar system, was also the largest member (diameter $\sim 2300 \mathrm{~km}$ ) of this new population of objects. Extrapolation of the TNO size distribution to the upper diameter range, showed that a few additional large TNOs (diameter $\geq 1000 \mathrm{~km}$ ) should exist in the Kuiper Belt. Subsequently, survey programs led to the discovery of bodies such as Quaoar $(\sim 850 \mathrm{~km})$, Sedna $(\sim 1500 \mathrm{~km})$, Orcus $(\sim 950 \mathrm{~km})$, and more recently $2003 \mathrm{EL}_{61}(\sim 1200 \mathrm{~km}), 2005 \mathrm{FY}_{9}(\sim 1500 \mathrm{~km})$, and 2003 UB $_{313}(\sim 2600 \mathrm{~km})$, (diameter estimates from Spitzer, Stansberry et al. 2007). In addition to the constraints they imposed on the accretion and collision processes in the Kuiper Belt, the large physical diameter of these TNOs make them sufficiently bright at optical wavelengths to carry out a detailed characterization of their surface composition from the Earth distance. The discovery of 2003 UB $_{313}$ (named 136199 Eris), a transneptunian object possibly bigger than Pluto (Brown et al. 2005) and whose semi-major orbital axis is almost twice as large, triggered lots of discussion about what type of solar system objects should be considered a planet. As a result, the IAU adopted a resolution stating that both TNOs Eris and Pluto are now members of a new category of solar system objects, the "dwarf planets", together with Ceres, the largest main-belt asteroid.

^ Based on observations obtained at the European Southern Observatory Very Large Telescope, Cerro Paranal, Chile. Program 275.C-5048(D).
136199 Eris is a scattered TNO orbiting the Sun at a very large distance (aphelion $\sim 98 \mathrm{AU}$ ) and on a highly inclined orbit $\left(44^{\circ}\right)$ whose dynamical characteristics follow that of a "detached transneptunian object", i.e. with pericenter decoupled from Neptune (Gladman et al. 2007). The first diameter estimation was determined by Bertoldi et al. (2006) by combining optical brightness measurements with millimeter wavelength observations. Based on the measured thermal emission, Bertoldi et al. derived a diameter of $3000 \pm 300 \mathrm{~km}$ and a visible albedo of $0.60 \pm 0.10$. Using the Hubble Space Telescope, Brown et al. (2006a) obtained a direct estimate of Eris' diameter of $2400 \pm$ $100 \mathrm{~km}(\sim 5 \%$ larger than Pluto) and derived an albedo of $0.86 \pm$ 0.07 (Pluto's albedo $\sim 0.6$ ).

Its near-infrared reflectance spectrum obtained at Gemini (Brown et al. 2005) shows strong similarities with the spectra of TNOs such as Pluto and $2005 \mathrm{FY}_{9}$, that is, strong absorption features characteristic of the presence of methane $\left(\mathrm{CH}_{4}\right)$ ice on the surface. But contrarily to Pluto, Brown et al. report moderate wavelength shifts for the central wavelengths of the $\mathrm{CH}_{4}$ bands, suggesting that methane ice is mostly found in its pure form on the surface of Eris, instead of being dissolved in a matrix of nitrogen $\left(\mathrm{N}_{2}\right)$ ice. Licandro et al. (2006) used the $4.2 \mathrm{~m}$ William Herschel Telescope to obtain a visible spectrum of Eris. They report stronger bands of methane ice than seen for Pluto, as well as a small wavelength shift around $0.89 \mu \mathrm{m}$ that they attribute to the presence of methane diluted in $\mathrm{N}_{2}$. By analogy with Pluto and Triton we could also expect ices such as $\mathrm{N}_{2}$ and $\mathrm{CO}$ (Schäller $\&$ Brown 2007) or $\mathrm{CO}_{2}$ to be present on the surface of Eris, but no detection has been made to date.

In this paper we report the analysis of new visible and near infrared spectra of the unresolved binary system composed of Eris and its small satellite (Brown et al. 2006b) using the TNG 
(Canary Island) and ESO-VLT (Chile) facilities. Radiative transfer models applied to our data allow us to investigate in greater details the composition, properties and distribution of the ices present on the surface of the largest transneptunian object known to date.

\section{Observations and data reductions}

ESO Director Discretionary Time (program 275.C-5048(D)) was allocated to observe the large trans-neptunian object $2003 \mathrm{UB}_{313}$, with the ESO-VLT (Very Large Telescope). The TNG telescope (Telescopio Nazionale Galileo), La Palma Observatory, was also used to obtain visible and near infrared spectra. These observations led to a complete set of spectroscopic data covering the visible and near infrared wavelength domain.

We used the SINFONI instrument (Spectrograph for INtegral Field Observations in the Near Infrared), installed at the $8 \mathrm{~m}$ "Yepun" ESO Very Large Telescope at Paranal Observatory, to collect $H$ - and $K$ - band spectra of Eris. SINFONI is an image slicer integral field spectrometer (Eisenhauer et al. 2003; Bonnet et al. 2004) whose field of view is split into 32 image-slitlets which reflect onto small plane mirrors before being re-directed toward the selected grating. The 32 spectra are then re-imaged on a $2048 \times 2048$ pixels Hawaii $2 \mathrm{RG}$ [1-2.5] $\mu$ m near-infrared detector. Although SINFONI is fed with an Adaptative Optics (AO) system, it can also be used in "no-AO" mode for seeing limited observations. 2003 UB $_{313}$ was observed in this mode on October 18, 2005, from 4 to 6 UT (airmass ranging from 1.07 to 1.14), under excellent meteorological conditions: photometric night, constant seeing at $0.6^{\prime \prime}$. We used the $\mathrm{H}+\mathrm{K}$ spectral grating (spectral resolution of 1500) covering both $H$ and $K$ bands simultaneously, and a plate scale of 250 mas/spaxel $\left(8^{\prime \prime} \times 8^{\prime \prime} \mathrm{FoV}\right)$, for a total integration time of two hours $(12 \times$ $600 \mathrm{~s}$ exposures). The solar analog HD 11532 (G5) was observed at similar airmass before and after the object, using the same instrumental setting. These observations provided the calibrations that were needed to correct our spectra from the solar response and telluric absorption features. Basic standard reduction was applied to the image set, i.e. bad pixel and flat field corrections. Xe-Ar-Kr lamps were used for wavelength calibration, and a wavelength map was computed to derive a direct correspondence between pixel position and wavelength. The determination of the orientation and position of each spectrum on the detector permits to reconstruct an image-cube of the original field of view. The object was dithered at different positions across the field in order to record the contribution of the sky background. Successive frames were then subtracted to correct our images from background contamination. The data were reduced using the Max Planck Institut für Extraterrestrische Physik (MPE) data reduction package, while wavemaps for the $\mathrm{H}+\mathrm{K}$ grating were obtained from the ESO SINFONI pipeline. The spectra were extracted from the individual data cubes using QFitsView, the 3D-visualisation tool developed at the MPE for SINFONI (http: //www . mpe .mpg.de/ ott/QFitsView). The individual spectra were then corrected from the remaining bad pixels and divided by the solar analog spectra. They were then combined to improve the signal-to-noise ratio (SNR) of the final product.

Spectral coverage in the visible and $J$-band ranges were obtained at the $3.8 \mathrm{~m}$ TNG telescope located in La Palma, Canary Islands, Spain. On August 30, 2005, we used the DOLORES visible spectrograph (Device Optimized for the LOw RESolution) with a slit width of $1.5^{\prime \prime}$ and the LR-R grism mode (resolution of 500) to obtain 50 min of integration time on target at an airmass of 1.2. A near infrared spectrum was also recorded on August 31, 2005 at an airmass of 1.25 using the Near Infrared Camera Spectrometer (NICS) equipped with the AMICI prism and a 1.5 -arcsec slit width. This instrument provides a low spectral resolution of $\sim 35$, almost constant throughout the 0.8-2.5 $\mu \mathrm{m}$ range. The Landolt (SA) 93-101 solar analog star was observed with both instruments to correct our visible and near-infrared spectra from the atmospheric absorption features and solar color. As seen in Fig. 1, we only consider the $0.8-1.3 \mu \mathrm{m}$ subset of the NICS data to connect our spectra between the visible (DOLORES) and the $H+K$ (SINFONI) bands. We applied the standard reduction procedure for visible and near infrared spectroscopy described by Fornasier et al. (2004). An average bias was created and subtracted from all the spectra, which were then divided by a normalized flat field, calibrated in wavelength and divided by the solar analog's spectrum.

\section{Surface modeling}

As shown in Fig. 1, the near-infrared reflectance spectrum of Eris resembles, at the first order, the spectrum of Pluto and $2005 \mathrm{FY}_{9}$ (Brown et al. 2007). The spectra of all three objects are dominated by the spectral signatures of methane ice, although the absorption bands on Eris appear to be stronger than in the case of Pluto. A weak and narrow absorption at 1.689 micron can also be seen in the near-infrared part of the spectrum. This band is not present in the spectrum of methane diluted in nitrogen (Grundy et al. 2002; Quirico et al. 1996) and is attributed from laboratory spectra to pure methane ice at temperatures lower than $60 \mathrm{~K}$. At lower wavelengths, the visible spectrum of Eris shows signatures around 0.73 and $0.89 \mu \mathrm{m}$, also diagnostic of methane ice (Grundy et al. 2002). However, the poor SNR of our data in this range hampers precise measurements of the depth and central wavelength of the $\mathrm{CH}_{4}$ bands.

Despite the good quality of the SINFONI data, no signature from ice other than methane can definitively be seen in our spectra. The characteristic near-IR absorption features of $\mathrm{CO}_{2}, \mathrm{CO}$ or $\mathrm{N}_{2}$, all expected to be present at these large heliocentric distances (Shäller \& Brown 2007), are not detected in our spectrum of Eris. Nevertheless, the medium spectral resolution $(R \sim 1500)$ of the VLT/SINFONI $H+K$-band spectrum, associated with the visible and $J$-band coverage obtained at TNG, permit to carry out a quantitative analysis of the compositional and physical properties of Eris surface and search for indirect evidence of ices other than methane. In order to estimate the temperature, particle size and mixing ratios of the compounds present on Eris, we first adjusted the different parts of the spectrum using photometric measurements of Brown et al. (2005). We then applied two spectral modeling methods (Hapke 1981; Shkuratov et al. 1999) covering the visible and near-infrared range of our spectra normalized to the two extreme albedo values that have been reported to date 0.6 (Bertoldi et al. 2006) and 0.86 (Brown et al. 2005). The optical constants of the ices come from Quirico \& Schmitt (1997) for pure methane ice (starting at $0.7 \mu \mathrm{m}$ ), from Grundy \& Schmitt (1998) for crystaline water ice, and from Quirico et al. (1996) for $\beta$-nitrogen ice. These optical constants were obtained with a temperature of $40 \mathrm{~K}$. Optical constants of ice tholin come from Khare et al. (1993). Ice tholin, a product of irradiation of ices, was introduced in the model to account for the reddish slope in the visible.

Adopting an albedo of 0.86 (Fig. 1), the best fit across our wavelength domain was obtained with an assemblage of essentially large icy grains distributed over two geographically 


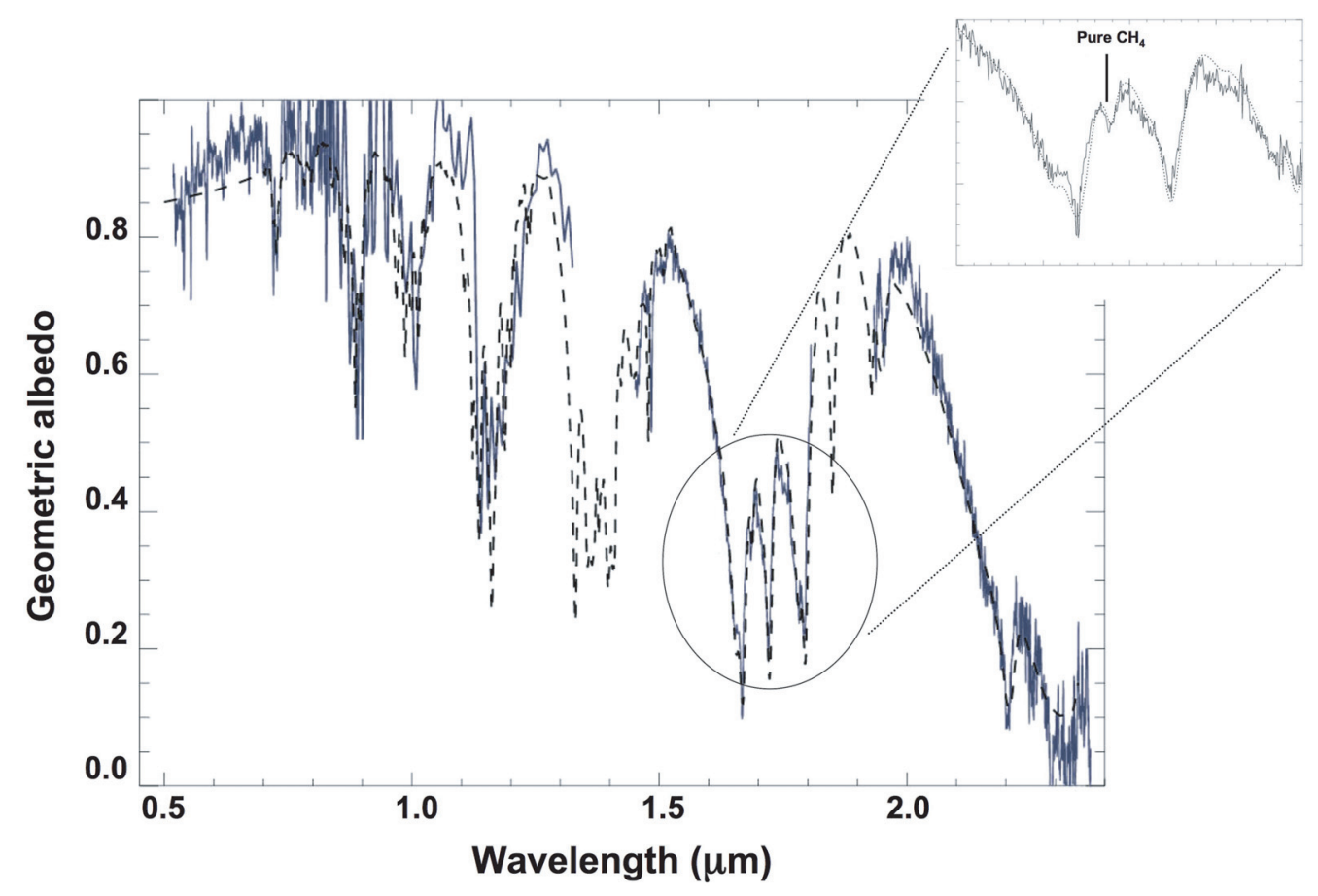

Fig. 1. Visible and near-infrared spectrum of 2003 UB313. The dotted line represents the best data fit obtained using Hapke modeling for a 0.86 geometric albedo value. The model requires large amounts of methane ice on the surface and introducing $\mathrm{N}_{2}$ ice (with large grain sizes) improves significantly the quality of the fit (see text). The surface of Eris can be modeled by two geographical areas of distinct composition. About half of its surface appears to be covered with pure methane ice ( $1 \mathrm{~mm}$ grain size). The remaining area corresponds to an intimate mixture of pure methane ice (14\%, $1 \mathrm{~mm}$ grain size), nitrogen ice (33\% with $\sim 2 \mathrm{~cm}$ grain size), crystalline water ice ( $2 \%, 0.1 \mathrm{~mm}$ grain size) and ice tholin $(2 \%, 10 \mu \mathrm{m}$ grain size). The insert box at top right provides a detailed zoom of the spectral region showing the absorption band of pure methane ice, which appears to be present in large amounts on the surface of Eris.

segregated zones. The first area consists of pure methane ice only, covering from 49 to $52 \%$ of the total surface of Eris for the Hapke and Shkuratov models respectively. The second area appears to be composed of an intimate mixture of pure methane ice ( $14 \%$ or $11 \%$, depending on the model), $\mathrm{N}_{2}$ ice (33\% or $26 \%$ ) with large $\sim 2 \mathrm{~cm}$ grain size, crystalline water ice ( $2 \%$ or $10 \%)$ and ice tholin ( $2 \%$ or $1 \%)$. In addition to returning similar abundances, the two modeling methods (Hapke or Shkuratov) require also similar large grain sizes to optimize the fit. The goodness of the fit has been evaluated from its $\chi^{2}$ value over the $2.0-2.3 \mu \mathrm{m}$ region, which corresponds to the location of the strong absorption band of $\mathrm{N}_{2}$. The $\chi^{2}$ value is decreased by $\sim 40 \%$ (from 10.2 to 6.0) when $\mathrm{N}_{2}$ is present in the model. The "reduced- $\chi^{2}$ ", is itself improved by $\sim 50 \%$, from 4.00 without $\mathrm{N}_{2}$, to 2.38 with $\mathrm{N}_{2}$. Figure 2 shows the comparison between the models in both cases, and the improvement made to the fit when $\mathrm{N}_{2}$ is considered.

For an albedo of 0.6, the lower reflectivity and optimum fit are reached by increasing slightly the abundances of water ice and ice tholin at the detriment of methane and nitrogen ices, which are the brightest compounds in our model. Similarly to the previous case, Fig. 3 shows an improvement of the fit in the $2.0-2.3 \mu \mathrm{m}$ region by adding $\mathrm{N}_{2}$ to the model (the $\chi^{2}$ is reduced by $70 \%$, from $\sim 13.4$ without $\mathrm{N}_{2}$, to $\sim 4.1$ with $\mathrm{N}_{2}$, while the "reduced- $\chi$ " " is improved by $5 \%$, from 1.47 without $\mathrm{N}_{2}$, to 1.40 with $\mathrm{N}_{2}$ ).

The reduced- $\chi^{2}$ shows that, regardless of the presence of $\mathrm{N}_{2}$ on Eris, our models provide a better fit to the data for the highest of the two albedos considered. Introducing $\mathrm{N}_{2}$ in our models improves the fit for both albedos, although the improvement is much lower if we consider an albedo of 0.6 rather than 0.86 .
Using infrared measurements made with Spitzer, Stansberry et al. (2007) derived a value around 0.7 for the albedo of Eris, which corresponds to an intermediate solution between the cases reported in Figs. 2 and 3. By comparison, although $\mathrm{N}_{2}$ ice dominates the surface of Pluto, its band at 2.15 micron is only detected from the shallow tip of its absorption feature in the downward slope of the methane band. The need for spectral modeling to test its presence on Eris, for which $\mathrm{N}_{2}$ seems to be much less predominant than on Pluto and whose spectrum has a lower SNR, is therefore not surprising.

As discussed above, most of the methane on Eris appears to be present in its pure, undiluted form. Table 1 shows the comparison of the positions of the main absorption bands of $\mathrm{CH}_{4}$ measured in our spectra with their expected positions in the case of pure methane ice and methane ice diluted in $\mathrm{N}_{2}$. According to Quirico \& Schmitt (1997) the dilution of methane in $\mathrm{N}_{2}$, even in small percentages, produces wavelength shifts of the order of 4 to $6 \mathrm{~nm}$. The spectral resolution of the VLT instrument and accuracy of its wavelength calibration are such that we can measure wavelength shifts of the order of $1 \mathrm{~nm}$. This can be verified by measuring the position of the weak methane absorption band visible in our spectra at $1.689 \pm 0.001 \mu \mathrm{m}$ (Fig. 1), which is characteristic of pure methane ice, and compare it to the position of $1.6894 \pm 0.0002 \mu \mathrm{m}$ obtained from laboratory measurements by Quirico \& Schmitt (1997). While the vast majority of methane ice is found in its pure form over the surface of Eris, we should consider that a few percents could still be diluted in $\mathrm{N}_{2}$ and remain undetected in our data. Another explanation for the absence of wavelength shift for methane ice could be that the abundance of methane diluted in $\mathrm{N}_{2}$ is at the contrary very high, resulting in the presence of large patches of methane, rather than 


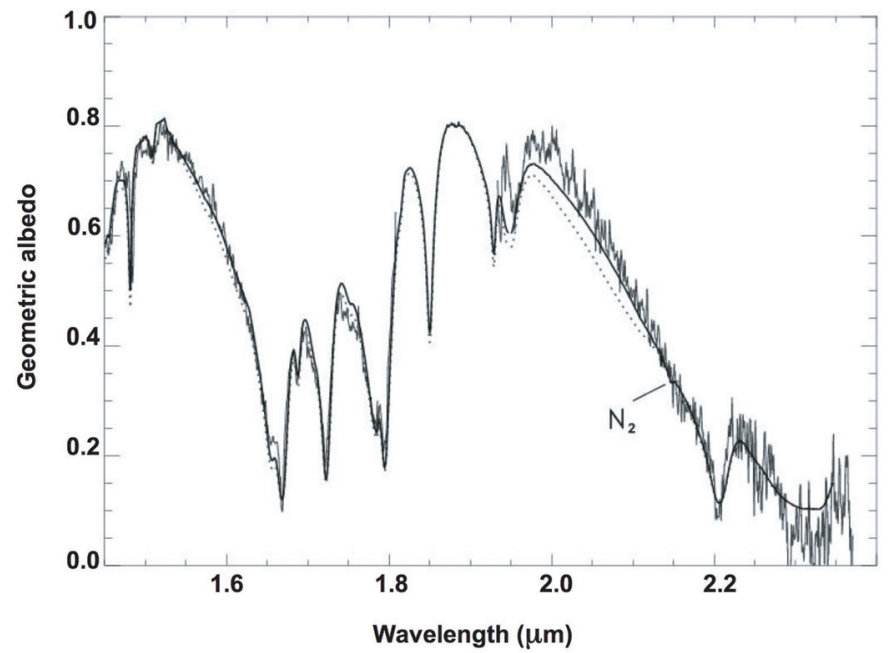

Fig. 2. $H+K$ band spectra of Eris and best fit Hapke model (solid curve) obtained for an albedo of 0.86 . The best model is obtained by mixing pure methane ice with an intimate mixture of ice tholin and methane, nitrogen and crystalline water ices. The same model without $\mathrm{N}_{2}$ is shown for comparison (dotted curve) and clearly deviates from the data, demonstrating that the presence of nitrogen improves significantly the spectral modeling results.

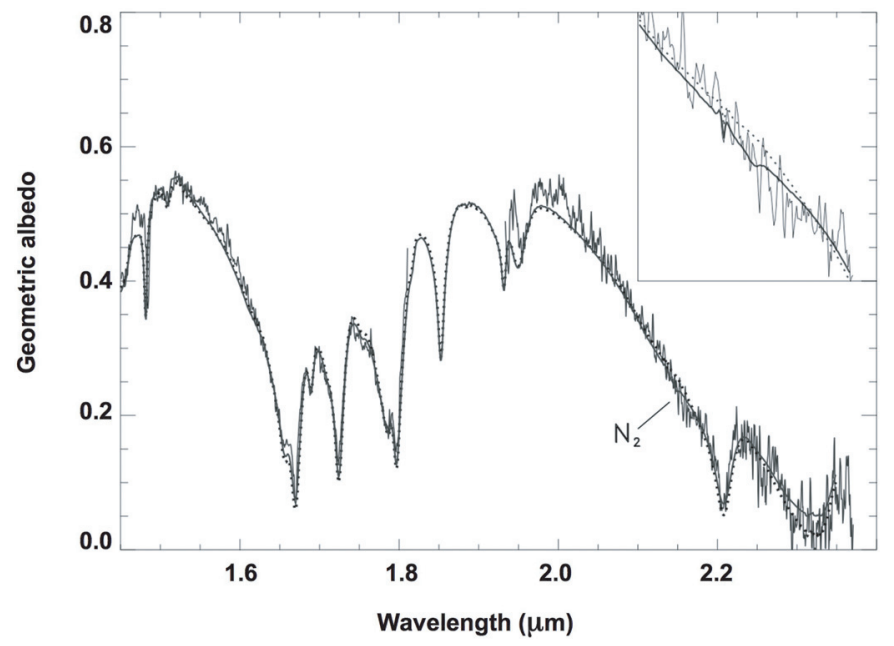

Fig. 3. Same $H+K$ band spectra of Eris and best fit model (solid curve) than before, but this time for an albedo of 0.6. The same model without $\mathrm{N}_{2}$ is shown for comparison (dotted curve). The insert box shows a zoom of the spectral region corresponding to the $2.15 \mu \mathrm{m}$ absorption band of $\mathrm{N}_{2}$. Although this band is not directly seen in our data, introducing nitrogen in our model returns a better fit to our spectroscopic data (see text).

being diluted in monomer state (Quirico \& Schmitt 1997). The large grain size returned by the models would also support this alternative hypothesis.

\section{Conclusions}

Pluto and Eris have lots in common: they are the two largest TNOs known to date, sustain nearly similar physical diameters, have both captured a satellite (although Eris' satellite is much smaller than Charon and has probably undergone different formation/capture mechanisms), and their surface composition appears to be almost identical, at least at a first order. Due to the highly eccentric orbit of Eris (perihelion at 37.8 AU; aphelion at 97.5 UA) we can expect Eris to sustain a thin atmosphere close to perihelion, which freezes out onto the surface when the
Table 1. Comparison of the central wavelength (in micron) of methane ice bands measured in our spectrum of Eris (left column) with the position of the same bands reported from laboratory experiments for the two cases of pure (middle column) and diluted (right column) methane ice.

\begin{tabular}{lcc}
\hline \hline Data & ${\text { Pure } \mathrm{CH}_{4}{ }^{a}}$ & Diluted $\mathrm{CH}_{4}{ }^{a}$ \\
\hline $1.484 \pm 0.001$ & 1.4850 & - \\
$1.656 \pm 0.001$ & 1.6575 & - \\
$1.671 \pm 0.001$ & 1.6697 & 1.6657 \\
$1.689 \pm 0.001$ & 1.6894 & none \\
$1.723 \pm 0.001$ & 1.7242 & 1.7198 \\
$1.787 \pm 0.001$ & 1.7873 & - \\
$1.797 \pm 0.001$ & 1.7967 & 1.7921 \\
$1.856 \pm 0.001$ & 1.8576 & - \\
$1.938 \pm 0.001$ & 1.9374 & - \\
$2.207 \pm 0.003$ & 2.2077 & 2.2024 \\
$2.377 \pm 0.004$ & 2.3789 & 2.3729 \\
\hline
\end{tabular}

${ }^{a}$ Quirico \& Schmitt (1997).

dwarf planet is located at larger heliocentric distances (orbital period 560 years). The high geometric albedo measured for Eris is possibly the result of fresh material being periodically redeposited onto the surface. Spectral modeling of the high quality data obtained with SINFONI at ESO-VLT provides some evidences that $\mathrm{N}_{2}$ is likely to be also present on the surface of Eris, covering about $30 \%$ of the total area, while methane is mainly found in its pure form. Our models show that the introduction of $\mathrm{N}_{2}$ ice improves the fit in the $2.0-2.3 \mu \mathrm{m}$ region, while water ice is needed to reproduce the envelope of our spectra. The collection of higher SNR $K$-band spectra, where lies the characteristic absorption band of nitrogen at $2.15 \mu \mathrm{m}$, is needed to unambiguously confirm the presence of $\mathrm{N}_{2}$ ice on Eris.

Acknowledgements. We would like to thank Stefan Gillessen (MPE) for his help with the SINFONI pipeline, as well as the Paranal and TNG Science Operations staff for their support, in particular M. Hartung and J. Navarrete (ESO) who obtained the SINFONI data in service mode. We also thank the referee, M. E. Brown, for his constructive comments, which helped improve the quality of this manuscript.

\section{References}

Bertoldi, F., Altenhoff, W., Weiss, A., Menten, K. M., \& Thum, C. 2006, Nature, 439,563

Bonnet, H., Abuter, R., Baker, A., et al. 2004, The ESO Messenger, 117, 17

Brown, M. E., Trujillo, C. A., \& Rabinowitz, D. L. 2005, ApJ, 635, L97

Brown, M. E., Schaller, E. L., Roe, H. G., Rabinowitz, D. L., \& Trujillo, C. A. 2006a, ApJ, 643, L61

Brown, M. E., van Dam, M. A., Bouchez, A. H., et al. 2006b, ApJ, 639, L43

Brown, M. E., Barkume, K. M., Blake, G. A., et al. 2007, AJ, 133, 284

Eisenhauer, F., Abuter, R., Bickert, K., et al. 2003, SPIE, 4841, 1548

Fornasier, S., Dotto, E., Barucci, M. A., \& Barbieri, C. 2004, A\&A, 422, L43

Gladman, B., Marsden, B. G., \& VanLaerhoven, C. 2007, in Kuiper Belt book, ed. M. A. Barucci et al. (Tucson: Univ. Arizona Press), in press

Grundy, W. M., \& Schmitt, B. 1998, J. Geophys. Res., 103, 25809

Grundy, W. M., Schmitt, B., \& Quirico, E. 2002, Icarus, 155, 486

Hapke, B. 1981, JGR, 86 B4, 3039

Jewitt, D., \& Luu, J. 1993, Nature, 362, 730

Jewitt, D., Luu., J., \& Trujillo, C. 1998, AJ, 115, 2125

Khare, B. N., Thompson, W. R., Cheng, L., et al. 1993, Icarus, 103, 290

Licandro, J., Grundy, W. M., Pinilla-Alonso, N., \& Leisy, P. 2006, A\&A, 458, L5

Luu, J., Marsden, B. G., Jewitt, D., et al. 1997, Nature, 387, 573

Quirico, E., \& Schmitt, B. 1997, Icarus, 127, 354

Quirico, E., Schmitt, B., Bini, R., \& Salvi, P. R. 1996, Planet. Space Sci., 44, 973

Schäller, E. L., \& Brown, M. E. 2007, ApJ, 659, L61

Shkuratov, Y., Starukhina, L., Hoffmann, H., \& Arnold, G. 1999, Icarus, 137, 235

Stansberry, J., Grundy, W., Brown, M., et al. 2007, in Kuiper Belt book, ed. M. A. Barucci et al. (Tucson: Univ. Arizona Press), in press 